\title{
AVIFAUNA DEL BOSQUE CHAQUEÑO ORIENTAL DE LA PROVINCIA DE CÓRDOBA, ARGENTINA
}

\section{BIRD FAUNA OF THE ORIENTAL CHACO FOREST OF CÓRDOBA PROVINCE, ARGENTINA}

\author{
Liliana Giraudo ${ }^{1}$, Maura Kufner ${ }^{2}$, Ricardo Torres ${ }^{1}$, Daniela Tamburini ${ }^{2}$, Verónica Briguera ${ }^{2}$ y Gregorio Gavier ${ }^{1}$
}

\section{Resumen}

El Chaco -hábitat reproductivo de aves migratorias- tiene mayor prioridad de conservación en Sudamérica. Su estacionalidad hídrica típica influye en la disponibilidad de recursos, así como en la composición y organización de avifauna. Sobre éstas inciden la fragmentación y reemplazo del bosque por expansión agrícola. Esta contribución proporciona la composición y riqueza de avifauna en remanentes del Chaco Oriental de Córdoba y realiza observaciones ecológicas sobre gremios tróficos, estatus de residencia y estado poblacional, a fin de interpretar su situación actual y promover su conservación. Dichos relictos se ubican entre la Reserva de Mar Chiquita y las Sierras del Norte, alternando con bosque secundario, parque y pastizales producidos por tala, y con parcelas cultivadas. Entre invierno de 2000 y otoño de 2002 se relevaron estacionalmente las aves en puntos fijos distribuidos al azar. Se detallaron: ubicación sistemática, nombre vulgar, estado poblacional, estatus de residencia y gremio trófico. Se hallaron un número elevado de especies (127) y entidades interesantes: amenazada (Harpyhaliaetus coronatus), en recuperación (Ortalis canicollis), acuáticas y "plagas agrícolas", acorde con la variedad de fisonomías y condiciones ecosistémicas del área. Las especies permanentes fueron mayoría expresándose su adaptación al bosque en la diversidad alimentaria. Las alteraciones del hábitat provocaron un cambio de $24 \%$ de especies antiguas permanentes, a ocasionales. Las migrantes estivales -coherentemente con la disponibilidad de recursos- triplicaron las invernales. Del gremio insectívoro participaron especies permanentes, migrantes y ocasionales, acorde a provisión de insectos en bosque subtropical semiárido. La biodiversidad remanente de aves chaqueñas aconseja su conservación.

Palabras clave: Chaco, Bosque relictual, Avifauna, Composición, situación y características funcionales

\section{Abstract}

The Chaco -reproductive habitat of migratory birds - has a great conservation priority in South America. Its typical seasonality influences the availability of resources, and the composition and organization of avifauna. Fragmentation and substitution of the forest for agricultural expansions, impact on these characteristics. This contribution provides the composition and richness of avifauna in relicts of the Oriental Chaco of Córdoba, and carries out ecological observations on trophic guilds, residence and conservation status, in order to interpret its current situation and to promote conservation. These forest remnants located between the Reserve of Mar Chiquita and Sierras del Norte, alternate with secondary forest, park and grassland, and cultivated parcels. From the 2000 winter to the 2002 autumn birds were observed seasonally in fixed points distributed at random. Characteristics detailed were: systematic classification, common name, population state, residence status and trophic guild. A high number of species (127) and interesting entities were found, according with the variety of ecosystem physiognomies and conditions in the area: threatened species (Harpyhaliaetus coronatus), in recovery (Ortalis canicollis), aquatic birds and "agricultural plagues". Permanent or resident species were the majority and their adaptation to the forest was expressed in diverse alimentary categories. Habitat alterations influenced a change of $24 \%$ in old permanent to occasional species. The spring-summer migrants -according to seasonal resources- tripled the winter ones. Permanent, migrants and occasional species participated in the insectivorous guild, according to a good provision of insects in semi-arid subtropical forest. Remainder biodiversity and functions of avifauna recommend the conservation of the Chaco forest in Córdoba.

Key words: Chaco, Relict forest, Birds Composition, Situation and Functional Characteristics 


\section{Introducción}

El Chaco se extiende en el S de Bolivia, W de Paraguay y el $\mathrm{N}$ de Argentina, con límite austral en el Norte de la provincia de Córdoba; es considerado una de las regiones con mayor prioridad de conservación en América del Sur (Beissinger et al., 1996). Su vegetación dominante, el bosque xerófilo caducifolio, se extiende en llanuras y serranías bajas, también con diferentes biomas neotropicales: palmares, sabanas y estepas arbustivas (Cabrera \& Willink, 1980). Florística y faunísticamente esta región posee características propias y pocos endemismos. Los bosques semiáridos chaqueños constituyen hábitat de reproducción de muchas aves migrantes neotropicales y neárticas (Codesido \& Bilenca, 2004). A mediados del siglo pasado se citaban más de 200 especies de aves chaqueñas (Olrog, 1963), en una basta planicie boscosa sin barreras físicas para el flujo aviar (Short, 1975). La avifauna del Chaco está compuesta por ensambles de distintas especies (Brooks, 1997), muchas distribuidas fuera de la región.

En la actualidad el Chaco está muy alterado por la explotación forestal y rápidamente modificado por el avance de la frontera agrícola, especialmente de soja. Al reemplazarse el bosque nativo por cultivos o potreros, se simplifica la estructura del hábitat, influyendo en la diversidad de aves y la extinción local de especies (Morello \& Matteucci, 1999; Albarece \& Antelo, 2000).

No obstante, se considera que el conocimiento ecológico y funcional de los ambientes chaqueños es aún inconcluso y superficial (Chani et al., 1998). Existen listas regionales como la de Short (1975) y la de Nores (1996) en Córdoba, que necesitan ser actualizadas. Asimismo, hay evidencia respecto a la marcada estacionalidad hídrica del Chaco y su influencia en la disponibilidad de recursos y en los gremios de aves, cuya variación -al menos desde el punto de vista trófico- se relacionaría con el ingreso estival de especies migrantes diferentes a las residentes (Capurro \& Bucher, 1988; Codesido \& Bilenca, 2004).

El objetivo de esta contribución es proporcionar la composición y riqueza específica de la avifauna hallada en remanentes de bosque del Chaco Oriental de Córdoba, Argentina, así como realizar observaciones de características ecológicas sobre gremios tróficos, estatus de residencia y estado poblacional. Ello, con el fin de mejorar la interpretación de su situación actual y destacar la importancia de conservar la biodiversidad chaqueña remanente.

\section{Material y Método}

Área de estudio. El área comprende relictos de bosque que se extienden entre la Reserva Bañados del Río Dulce-Laguna de Mar Chiquita y las Sierras del Norte.
Los dominantes fisonómicos son: Aspidosperma quebracho-blanco, Celtis tala, Lithraea ternifolia, Schinopsis sp., Acacia sp. Prosopis sp. y Geoffroea decorticans. Ésta y el cardón Stetsonya coryne forman bosquecillos en suelos salinos. Fragmentos del hábitat chaqueño alternan con la fisonomía de parque de Schinopsis sp. y pastizales producto de tala selectiva, así como con parcelas en distinto grado de modificación entre bosque secundario y cultivos (Cabido \& Zak, 1999). Descripción detallada del área de estudio en Kufner et al. (2005).

La investigación se llevó a cabo en el interior de unidades homogéneas de bosque natural, seleccionadas en base a cartografía vegetal, resuelta a partir de la clasificación de una imagen Lansat (Zak \& Cabido, 2002). Entre invierno de 2000 y otoño de 2002 se muestrearon las especies de aves en un total de 12 puntos fijos distribuidos al azar, separados entre sí por no menos de $200 \mathrm{~m}$. Por estación, en cada punto, se realizaron 5 observaciones de 15 minutos cada una (Kufner et al., 1998; Albarece \& Antelo, 2000). En cada observación se registraron todas las especies vistas y oídas en un radio aproximado de 15 m. Se llevaron a cabo en total 8 muestreos, cada uno tuvo una duración de 3 días, extendiéndose desde el amanecer hasta las 11 horas y desde las 16 horas hasta el anochecer.

La lista de especies se elaboró considerando su ubicación sistemática actualizada, incluyendo el nombre vulgar más frecuente en Argentina (Mazar Barnett \& Pearman, 2001; Narosky \& Yzurieta, 2003). Siempre que fue posible se consignó el estado poblacional de las especies, siguiendo las categorías publicadas a nivel internacional $(\mathrm{CI})$ y provincial $(\mathrm{P})$, tomando las de Birdlife International (2006) y la de Miatello (1994), respectivamente.

Se analizó la presencia estacional de las especies en el área, asignándoles categorías o estatus de residencia (Codesido \& Bilenca, 2004; AlmazánNúñez \& Navarro, 2006), según se describe a continuación: Permanente (Per): especie registrada en las cuatro estaciones; Estacional: especie registrada únicamente en estación húmeda estival (septiembremarzo) (Eest) o en estación seca invernal (AbrilAgosto) (Einv) y Ocasional (Oca): especie registrada sólo en una ocasión y/o en bajo número. Asimismo, se clasificaron las especies según la principal característica de su dieta en los siguientes gremios tróficos: O: Omnívoro, I: Insectívoro, G: Granívoro, C; Carnívoro, $\tilde{N}$ : Carroñero, F: Frugívoro, N: nectarívoro y H: Herbívoro (Sick, 1985; Leveau \& Leveau, 2004).

\section{Resultados}

Se relevó un total de 127 especies de aves pertenecientes a 33 familias, reunidas en 16 órdenes. Las aves clasificadas según gremios tróficos 
integraron alrededor del 49\% de insectívoras, $16 \%$ de omnívoras (incluyendo a F-I), 16\% de granívoras (incluyendo a G-F), 14\% de carnívoras (incluyendo a C-Ñ-I), $3 \%$ de carroñeras, menos de $2 \%$ de nectarívoros- insectívoros y sólo un herbívoro (Tabla 1).

En cuanto a su estatus de residencia, correspondió el $46 \%$ a especies de presencia permanente, $27 \%$ a las ocasionales, $19 \%$ a las estacionales de período estival húmedo (Eest) y $6 \%$ a las de período invernal seco (Einv). Del total, se identificaron 31 especies que cambiaron su estatus de permanente (Nores, 1996) a ocasional, principalmente. Además, la proporción de visitantes estivales triplicó a los invernales (Tabla 1).

Las especies insectívoras resultaron las mejor representadas en todas las categorías de residencia. Del gremio de insectívoros participaron tanto aves permanentes como ocasionales y migrantes y, en cada caso, con mayoría de integrantes. Por su parte, las aves de residencia permanente concentraron la mayor diversidad de categorías alimenticias (Tabla 1).

\section{$\underline{\text { Lista comentada de las especies }}$}

Clase AVES

Orden RHEIFORMES

Familia Rheidae

Rhea americana Lynch Arribálzaga y Holmberg 1878. (ñandú).

Estación observada: otoño.

Estado poblacional: CI: amenazado. P: Vulnerable

Observaciones generales: Se registraron dos pequeños grupos

únicamente en campos con perímetro alambrado.

\section{Orden TINAMIFORMES}

Familia Tinamidae

Crypturellus tataupa (Temmink) (tataupá común).

Estado poblacional: CI: preocupación menor.

Observaciones generales: Se identifica por su canto característico.

Más escuchado que visto.

Nothoprocta cinerascens (Burmeister) (inambú montaraz). Estado poblacional: CI: preocupación menor. P: Marcado retroceso numérico.

Nothura maculosa Conover (inambú común). Estación observada: verano, otoño e invierno Estado poblacional: CI: preocupación menor

Eudromia elegans Olrog (martineta común). Estación observada: primavera y otoño

Estado poblacional: CI: preocupación menor. P: Marcado retroceso numérico.

\section{Orden CICONIIFORMES}

Familia Ardeidae

Syrigma sibilatrix (Temmink) (chiflón).

Estación observada: otoño

Estado poblacional: CI: preocupación menor.

Observaciones generales: en relación con bañados próximos y pastizales, donde se alimenta.

Bubulcus ibis (Linnaeus) (garcita bueyera). Estación observada: Primavera

Estado poblacional: CI: Preocupación menor Observaciones generales: en relación con bañados próximos y pastizales, donde se alimenta.
Casmerodius albus $=$ Egretta alba (Gmelin) (garza blanca).

Estación observada: Invierno

Estado poblacional: CI: Preocupación menor

Observaciones generales: en relación con bañados próximos y

pastizales, donde se alimenta.

\section{Orden FALCONIFORMES \\ Familia Cathartidae}

Coragyps atratus (Bechstein) (jote cabeza negra).

Estación observada: Primavera, verano, otoño, invierno

Estado poblacional: CI: Preocupación menor

Observaciones generales: Integra el apéndice II de CITES

Cathartes aura (Molina) (jote cabeza colorada).

Estación observada: Primavera, verano, otoño, invierno

Estado poblacional: CI: Preocupación menor

Observaciones generales: Integra el apéndice II de CITES

Cathartes burrovianus Pelzeln (jote cabeza amarilla).

Estación observada: primavera, verano

Estado poblacional: CI: Preocupación menor.

Observaciones generales: escaso registro en la provincia.

Mencionado en el sur de Mar Chiquita, en las Salinas Grandes

(Torres \& Michelutti, 2001). Integra el apéndice II de CITES.

Sarcoramphus papa (Linnaeus) (jote real).

Estación observada: otoño.

Estado poblacional: CI: Preocupación menor

Observaciones generales: Registros escasos en Córdoba. Integra el apéndice II de CITES.

\section{Familia Accipitridae}

Elanus leucurus (Vieillot) (milano blanco).

Estación observada: otoño, invierno

Estado poblacional: CI: Preocupación menor

Observaciones generales: Integra el apéndice II de CITES.

Harpyhaliaetus coronatus (Vieillot) (águila coronada). Estación observada: otoño, invierno, verano Estado poblacional: CI: Vulnerable, En peligro; P: Marcado retroceso numérico

Observaciones generales: Integra el apéndice II de CITES

Buteo magnirostris (Sclater \& Salvin) (taguató común).

Estación observada: verano

Estado poblacional: CI: Preocupación menor

Buteo swainsoni Bonaparte (aguilucho langostero).

Estación observada: Otoño

Estado poblacional: CI: preocupación menor

Observaciones generales: Migrante en bandadas del Hemisferio

Norte (Nores, 1996).

Buteo polyosoma (Quoy \& Gaimard) (aguilucho común). Estación observada: Otoño, invierno

Estado poblacional: CI: Preocupación menor

Familia Falconidae

Polyborus plancus (Miller) (carancho).

Estación observada: Primavera, verano, otoño, invierno

Estado poblacional: CI: Preocupación menor

Observaciones generales: Integra el apéndice II de CITES

Polyborus chimango Vieillot (chimango).

Estación observada: Primavera, verano, otoño, invierno

Estado poblacional: CI: Preocupación menor

Observaciones generales: Integra el apéndice II de CITES

Spiziapteryx circumcinctus (Kaup) (halconcito gris).

Estación observada: Otoño, invierno

Estado poblacional: CI: Preocupación menor

Observaciones generales: Integra el apéndice II de CITES 
Falco femoralis Temmink (halcón plomizo).

Estación observada: Verano

Estado poblacional: CI: Preocupación menor. P: Marcado retroceso numérico

Observaciones generales: Integra el apéndice II de CITES

Falco sparverius Swainson (halconcito colorado).

Estación observada: Invierno

Estado poblacional: CI: preocupación menor

Observaciones generales: Integra el apéndice II de CITES

Orden GALLIFORMES

Familia Cracidae

Ortalis canicolis (Wagler) (charata).

Estación observada: Primavera, verano, otoño, invierno

Estado poblacional: CI: Preocupación menor. P: Vulnerable

Observaciones generales: su presencia en los bosques al W de Mar

Chiquita confirma la recuperación poblacional mencionada por

Nores (1996).

Orden GRUIFORMES

Familia Cariamidae

Chunga burmeisteri (Hartlaub) (chuña patas negras).

Estación observada: Primavera

Estado poblacional: CI: Preocupación menor. P: Marcado retroceso numérico

\section{Orden CHARADRIIFORMES}

Familia Charadriidae

Vanellus chilensis (Wagler) (tero común).

Estación observada: Primavera, verano, otoño

Estado poblacional: CI: Preocupación menor

Observaciones generales: relacionado con pastizales.

Orden COLUMBIFORMES

Familia Columbidae

Columba picazuro Temmink (paloma picazuró).

Estación observada: Primavera

Estado poblacional: CI: Preocupación menor

Columba maculosa Temmink (paloma manchada).

Estación observada: Primavera, verano, otoño, invierno

Estado poblacional: CI: Preocupación menor

Zenaida auriculata (Reichenbach) (torcaza).

Estación observada: Primavera, verano, otoño, invierno

Estado poblacional: CI: Preocupación menor

Observaciones generales: es la granívora con mayor número de individuos en este estudio. Considerada plaga agrícola a nivel provincial.

Columbina picui (Temmink) (torcacita común).

Estación observada: Primavera, verano, otoño, invierno

Estado poblacional: CI: Preocupación menor

Leptotila verreauxi (Salvadori) (yerutí común).

Estación observada: Primavera, verano, otoño, invierno

Estado poblacional: CI: Preocupación menor

Orden PSITTACIFORMES

Familia Psittacidae

Aratinga acuticaudata (Vieillot) ( calancate común)

Estación observada: Verano, otoño, invierno

Estado poblacional: CI: Preocupación menor

Myiopsitta monachus (Jardine \& Selby) (cotorra).

Estación observada: Primavera, verano, otoño, invierno

Estado poblacional: CI: Preocupación menor

Observaciones generales: considerada plaga agrícola a nivel provincial.

\section{Orden CUCULIFORMES}

Familia Cuculidae
Coccyzus melacoryphus Vieillot (cuclillo canela).

Estación observada: Primavera, verano

Estado poblacional: CI: Preocupación menor

Tapera naevia (Vieillot) (crespín).

Estación observada: Primavera, verano

Estado poblacional: CI:P preocupación menor

Observaciones generales: Más escuchado que visto.

Crotophaga ani Linnaeus (anó chico).

Estación observada: verano

Estado poblacional: CI: Preocupación menor

Observaciones generales: su presencia puede variar localmente con

los años (Nores, 1996).

Guira guira (Gmelin) (pirincho).

Estación observada: Primavera, verano, otoño, invierno

Estado poblacional: CI: Preocupación menor

Orden STRIGIFORMES

Familia Tytonidae

Tyto alba (J. E. Gray) (lechuza del campanario).

Estación observada: Otoño, invierno.

Estado poblacional: CI: Preocupación menor

\section{Familia Strigidae}

Otus choliba (Vieillot) (alicuco común).

Estación observada: otoño, invierno

Estado poblacional: CI: Preocupación menor

Glaucidium sp.

Estación observada: Otoño, invierno

Estado poblacional: CI: Preocupación menor

Observaciones generales: Nores (1996) reconoce dos especies, $G$.

brasilianum brasilianum (Gmelin) (caburé chico) y G. nanum

(King) (caburé grande), ambos de difícil identificación por su

similitud de apariencia y canto.

Athene cunicularia Olrog (lechucita de las vizcacheras).

Estación observada: Primavera, verano, otoño, invierno

Estado poblacional: CI: Preocupación menor. P: Marcado retroceso numérico

Strix rufipes Cherrie \& Reichenberger (lechuza bataraz)

Estación observada: verano

Estado poblacional: CI: Preocupación menor

Orden CAPRIMULGIFORMES

Familia Caprimulgidae

Podager nacunda Vieillot (ñacundá)

Estación observada: Primavera, verano

Estado poblacional: CI: Preocupación menor

Caprimulgus longirostris Olrog (atajacaminos ñañarca)

Estación observada: Otoño, invierno

Estado poblacional: CI: Preocupación menor

Caprimulgus parvulus Gould (atajacaminos chico)

Estación observada: Otoño, verano, primavera

Estado poblacional: CI: Preocupación menor

Hydropsalis brasiliana Vieillot (atajacaminos tijera común)

Estación observada: Verano

Estado poblacional: CI: Preocupación menor

Orden TROCHILIFORMES

Familia Trochilidae

Chlorostilbon aureoventris (d'Orbigny \& Lafresnaye) (picaflor

común).

Estación observada: Primavera

Estado poblacional: CI: Preocupación menor

Heliomaster furcifer (Shaw) (picaflor de barbijo). 
Estación observada: Primavera

Estado poblacional: CI: Preocupación menor

Orden PICIFORMES

Familia Bucconidae

Nystalus maculatus (Sclater) (durmilí).

Estación observada: Primavera, verano, otoño, invierno

Estado poblacional: CI: Preocupación menor

\section{Familia Picidae}

Melanerpes cactorum (d'Orbigny) (carpintero de los cardones)

Estación observada: Primavera, otoño

Estado poblacional: CI: Preocupación menor

Picoides mixtus (Wetmore) (carpinterito bataraz chico).

Estación observada: Verano, otoño, invierno

Estado poblacional: CI: Preocupación menor

Colaptes melanochloros Leybold (carpintero real).

Estación observada: Primavera, verano, otoño, invierno

Estado poblacional: CI: Preocupación menor

Colaptes campestris (Malherbe) (carpintero campestre)

Estación observada: Primavera, verano

Estado poblacional: CI: Preocupación menor

Orden PASSERIFORMES

Familia Dendrocolaptidae

Drymornis bridgesii (Eyton) (chinchero grande).

Estación observada: Invierno

Estado poblacional: CI: Preocupación menor

Lepidocolaptes angustirostris Esteban (chinchero chico). Estación observada: Primavera, verano, invierno

Estado poblacional: CI: Preocupación menor

\section{Familia Furnariidae}

Upucerthia certhioides (Wetmore \& Peters) (bandurrita chaqueña) Estación observada: Primavera, verano, invierno

Estado poblacional: CI: Preocupación menor

Furnarius rufus Cherrie \& Reichenberger (hornero). Estación observada: Primavera, verano, otoño, invierno Estado poblacional: CI: Preocupación menor

Furnarius cristatus Burmeister (hornerito). Estación observada: Verano

Estado poblacional: CI: Preocupación menor

Leptasthenura platensis Reichenbach (coludito copetón). Estación observada: Invierno

Estado poblacional: CI: Preocupación menor

Synallaxis frontalis Pelzeln (Pijuí Frente Gris).

Estación observada: Primavera, verano

Estado poblacional: CI: Preocupación menor

Synallaxis albescens Zimmer (pijuí cola parda).

Estación observada: Primavera, verano

Estado poblacional: CI: Preocupación menor

Cranioleuca pyrrophia (Vieillot) (curutié vientre blanco).

Estación observada: Invierno, primavera

Estado poblacional: CI: Preocupación menor

Asthenes baeri (Berlepsch) (canastero chaqueño).

Estación observada: Invierno

Estado poblacional: CI: Preocupación menor

Phacellodomus sibilatrix Sclater (espinero chico).

Estación observada: Otoño

Estado poblacional: CI: Preocupación menor
Coryphistera alaudina Burmeister (crestudo). Estación observada: Primavera, verano, otoño Estado poblacional: CI: Preocupación menor

Anumbius annumbi (Vieillot) (leñatero).

Estación observada: Otoño, primavera

Estado poblacional: CI: Preocupación menor

Pseudoseisura lophotes Parkes (cacholote castaño).

Estación observada: Primavera, verano, otoño, invierno

Estado poblacional: CI: Preocupación menor

Familia Formicariidae

Taraba major (Vieillot) (chororó).

Estación observada: Verano, invierno

Estado poblacional: CI: Preocupación menor

Thamnophilus caerulescens Berlepsch (choca común).

Estación observada: Primavera, verano, otoño, invierno

Estado poblacional: CI: Preocupación menor

\section{Familia Rhinocryptidae}

Rhinocrypta lanceolata (Geoffroy Saint-Hilaire) (gallito copetón). Estación observada: Otoño

Estado poblacional: CI: Preocupación menor. P: Marcado retroceso numérico

Familia Tyranidae

Camptostoma obsoletum (Temminck) (piojito silbón)

Estación observada: Otoño

Estado poblacional: CI: Preocupación menor

Elaenia parvirostris Pelzeln (fiofío pico corto).

Estación observada: Primavera, verano, otoño, invierno

Estado poblacional: CI: Preocupación menor

Serpophaga subcristata (Vieillot) (piojito común)

Estación observada: Primavera, verano

Estado poblacional: CI: Preocupación menor

Stigmatura budytoides Wetmore \& Peters (calandrita).

Estación observada: Primavera, verano, otoño, invierno

Estado poblacional: CI: Preocupación menor

Euscarthmus meloryphus Wied (barullero).

Estación observada: Verano, otoño

Estado poblacional: CI: Preocupación menor

Pyrocephalus rubinus (Boddaert) (churrinche).

Estación observada: Verano

Estado poblacional: CI: Preocupación menor

Agriornis murina d'Orbigny \& Lafresnaye (gaucho chico)

Estación observada: Invierno

Estado poblacional: CI: Preocupación menor

Observaciones generales: Migrante del sur argentino (Nores, 1996).

Xolmis irupero (Vieillot) (monjita blanca).

Estación observada: Primavera, verano, otoño, invierno

Estado poblacional: CI: Preocupación menor

Xolmis coronata (Vieillot) (monjita coronada).

Estación observada: Otoño, invierno

Estado poblacional: CI: Preocupación menor

Observaciones generales: Migrante del sur argentino (Nores, 1996).

Neoxolmis rubetra (Burmeister) (monjita castaña)

Estación observada: Primavera

Estado poblacional: CI: Preocupación menor

Observaciones generales: Migrante del sur argentino (Nores, 1996).

Knipolegus aterrimus Kaup (viudita común).

Estación observada: Verano, otoño, invierno 
Estado poblacional: CI: Preocupación menor

Knipolegus striaticeps d'Orbigny \& Lafresnaye (viudita chaqueña). Estación observada: Primavera, verano, invierno

Estado poblacional: CI: Preocupación menor

Myiarchus swainsoni Cabanis (burlisto pico pardo).

Estación observada: Primavera

Estado poblacional: CI: Preocupación menor

Pitangus sulfuratus Todd (benteveo común).

Estación observada: Primavera, verano, otoño, invierno

Estado poblacional: CI: Preocupación menor

Empidonomus aurantioatrocristatus d'Orbigny \& Lafresnaye (tuquito gris).

Estación observada: Primavera, verano

Estado poblacional: CI: Preocupación menor

Sublegatus modestus d'Orbigny \& Lafresnaye (suirirí pico corto). Estación observada: Verano

Estado poblacional: CI: Preocupación menor

Hirundinea ferruginea Hartert\& Goodson (birro común).

Estación observada: Otoño

Estado poblacional: CI: Preocupación menor

Machetornis rixosus (Vieillot) (picabuey).

Estación observada: Primavera

Estado poblacional: CI: Preocupación menor

Hemitriccus margaritaceiventer d'Orbigny \& Lafresnaye (mosqueta ojo dorado).

Estación observada: Otoño, invierno

Estado poblacional: CI: Preocupación menor

Myiophobus fasciatus Gould (mosqueta estriada).

Estación observada: Primavera, verano

Estado poblacional: CI: Preocupación menor

Tyrannus savana Vieillot (tijereta).

Estación observada: Primavera, verano

Estado poblacional: CI: Preocupación menor. P: Marcado retroceso numérico

Observaciones generales: Nidifica en la provincia en el período estival (Nores, 1996).

Pachyramphus polychopterus (Swainson) (anambé común).

Estación observada: Verano

Estado poblacional: P: Vulnerable

\section{Familia Phytotomidae}

Phytotoma rutila Vieillot (cortarramas).

Estación observada: Primavera, verano, otoño, invierno

Estado poblacional: CI: Preocupación menor

Observaciones generales: Única especie de Córdoba exclusivamente herbívora.

\section{Familia Hirundinidae}

Phaeprogne tapera (Vieillot) (golondrina parda).

Estación observada: Primavera, verano

Estado poblacional: CI: Preocupación menor

Observaciones generales: Nidifica en la provincia en el período estival (Nores, 1996).

Tachicyneta leucorrhoa (Vieillot) (golondrina ceja blanca).

Estación observada: Primavera

Estado poblacional: CI: Preocupación menor

Observaciones generales: Nidifica en la provincia en el período estival (Nores, 1996).

Progne modesta $=$ elegans Baird (golondrina negra).

Estación observada: Verano
Estado poblacional: CI: Preocupación menor

Observaciones generales: Nidifica en la provincia en el período estival (Nores, 1996).

Notiochelidon cyanoleuca d'Orbigny \& Lafresnaye (golondrina barranquera)

Estación observada: Primavera, verano

Estado poblacional: CI: Preocupación menor

Observaciones generales: Nidifica en la provincia en el período estival (Nores, 1996).

Familia Troglodytidae

Troglodytes aedon Berlepsch \& Leverkühn (ratona común).

Estación observada: Primavera, verano, otoño, invierno

Estado poblacional: CI: Preocupación menor

Familia Muscicapidae

Subfamilia Sylviinae

Polioptila dumicola (Vieillot) (tacuarita szul).

Estación observada: Primavera, verano, otoño, invierno

Estado poblacional: CI: Preocupación menor

Subfamilia Turdinae

Turdus amaurochalinus Cabanis (zorzal chalchalero).

Estación observada: Primavera, verano, otoño, invierno

Estado poblacional: CI: Preocupación menor. P: Marcado retroceso numérico

Turdus rufiventris Vieillot (zorzal colorado).

Estación observada: Verano

Estado poblacional: CI: Preocupación menor

\section{Familia Mimidae}

Mimus saturninus (Gould) (calandria grande)

Estación observada: Invierno, primavera

Estado poblacional: CI: Preocupación menor

Mimus triurus (Vieillot) (calandria real).

Estación observada: Otoño, invierno

Estado poblacional: CI: Preocupación menor

\section{Familia Motacilidae}

Anthus correndera Vieillot (cachirla común)

Estación observada: Verano, otoño

Estado poblacional: CI: Preocupación menor

\section{Familia Vireonidae}

Vireo olivaceus (Vieillot) (chiví común).

Estación observada: Primavera, verano

Estado poblacional: CI: Preocupación menor

Cyclarhis guajanensis (Vieillot) (juan chiviro).

Estación observada: Primavera, verano, invierno

Estado poblacional: CI: Preocupación menor

\section{Familia Emberizidae}

Subfamilia Parulinae

Parula pitiayumi (Vieillot) (pitiayumí).

Estación observada: Verano

Estado poblacional: Sin datos.

Geothlypis aequinoctalis (Vieillot) (arañero cara negra).

Estación observada: Primavera, verano

Estado poblacional: CI: Preocupación menor

Euphonia chlorotica d'Orbigny \& Lafresnaye (tangará común)

Estación observada: Otoño

Estado poblacional: CI: Preocupación menor

Subfamilia Thraupinae

Thraupis bonariensis Brodkorb (naranjero)

Estación observada: Primavera, verano, otoño, invierno

Estado poblacional: CI: Preocupación menor 
Subfamilia Cardinalinae

Saltator aurantiirostis Vieillot (pepitero de collar).

Estación observada: Primavera, verano, otoño, invierno

Estado poblacional: CI: Preocupación menor

Subfamilia Emberizinae

Paroaria coronata (Miller) (cardenal común).

Estación observada: Primavera, invierno

Estado poblacional: CI: Preocupación menor. P: Vulnerable

Observaciones generales: Integra el apéndice II de CITES.

Sporophila caerulescens (Vieillot) (corbatita común).

Estación observada: Verano, otoño

Estado poblacional: CI: Preocupación menor. P: Marcado retroceso numérico

Coryphospingus cucullatus Brodkorb (brasita de fuego).

Estación observada: Primavera, verano, otoño

Estado poblacional: CI: Preocupación menor. P: Marcado retroceso numérico

Saltatricula multiculor (Burmeister) (pepitero chico).

Estación observada: Primavera, verano, otoño

Estado poblacional: CI: Preocupación menor

Sicalis flaveola Sclater (jilguero dorado).

Estación observada: Primavera

Estado poblacional: CI: Preocupación menor

Sicalis luteola (meyen) (misto).

Estación observada: Primavera, verano, otoño

Estado poblacional: CI: Preocupación menor

Embernagra platensis d'Orbigny (verdón).

Estación observada: Otoño

Estado poblacional: CI: Preocupación menor

Poospiza melanoleuca (d'Orbigny \& Lafresnaye) (monterita cabeza negra).

Estación observada: Invierno, primavera

Estado poblacional: CI: Preocupación menor. P: Marcado retroceso numérico

Poospiza nigrorufa (d'Orbigny \& Lafresnaye) (sietevestidos)

Estación observada: Verano

Estado poblacional: CI: Preocupación menor

Poospiza torquata Todd (monterita de collar).

Estación observada: Verano, invierno

Estado poblacional: CI: Preocupación menor

Aimophila strigiceps (Gould) (cachilo corona castaña).

Estación observada: Primavera, verano, otoño, invierno

Estado poblacional: CI: Preocupación menor

Zonotrichia capensis (Wetmore \& Peters) (chingolo).

Estación observada: Primavera, verano, otoño, invierno

Estado poblacional: CI: Preocupación menor

Familia Icteridae

Sturnella superciliaris (Bonaparte) (pecho colorado)

Estación observada: Primavera, otoño

Estado poblacional: CI: Preocupación menor

Observaciones generales: Común en bañados (Nores, 1996).

Molothrus bonariensis (Gmelin) (tordo renegrido).

Estación observada: Primavera, verano, otoño

Estado poblacional: CI: Preocupación menor

Molothrus rufoaxillaris Cassin (tordo pico corto).

Estación observada: Primavera

Estado poblacional: CI: Preocupación menor
Molothrus badius (Vieillot) (tordo músico).

Estación observada: Primavera, verano, otoño

Estado poblacional: CI: Preocupación menor

Carduelis magellanica (Ridgeway) (cabecita negra común)

Estación observada: Primavera, verano

Estado poblacional: CI: Preocupación menor

Tabla 1. Gremios tróficos y estatus de residencia de las aves observadas en el Bosque Chaqueño

Oriental de Córdoba.

\begin{tabular}{ccc}
\hline ESPECIES & $\begin{array}{c}\text { GREMIO } \\
\text { TRÓFICO }\end{array}$ & $\begin{array}{c}\text { ESTATUS } \\
\text { DE } \\
\text { RESIDENCIA }\end{array}$ \\
\hline
\end{tabular}

\begin{tabular}{|c|c|c|}
\hline Rhea americana & $\mathrm{O}$ & $\mathrm{Oca}^{1}$ \\
\hline Crypturellus tataupa & $\mathrm{O}$ & Per \\
\hline Nothoprocta cinerascens & $\mathrm{O}$ & Per \\
\hline Nothura maculosa & $\mathrm{O}$ & Per \\
\hline Eudromia elegans & $\mathrm{O}$ & Per \\
\hline Syrigma sibilatrix & C-I & Oca \\
\hline Bubulcus ibis & C-I & Oca \\
\hline Casmerodius albus & $\mathrm{C}$ & Oca \\
\hline Coragyps atratus & $\tilde{\mathrm{N}}$ & Per \\
\hline Cathartes aura & $\tilde{\mathrm{N}}$ & Per \\
\hline Cathartes burrovianus & $\tilde{\mathrm{N}}$ & Eest \\
\hline Sarcoramphus papa & $\tilde{\mathrm{N}}$ & Oca \\
\hline Elanus leucurus & $\mathrm{C}$ & Einv \\
\hline Harpyhaliaetus coronatus & $\mathrm{C}$ & $\mathrm{Pe}$ \\
\hline Buteo magnirostris & $\mathrm{C}$ & Oca \\
\hline Buteo swainsoni & C-I & Oca \\
\hline Buteo polyosoma & $\mathrm{C}$ & Oca \\
\hline Polyborus plancus & $\mathrm{C}-\tilde{\mathrm{N}}-\mathrm{I}$ & Per \\
\hline Polyborus chimango & C-Ñ-I & Per \\
\hline Spiziapteryx & C-I & Ein \\
\hline circumncinctus & & \\
\hline Falco femoralis & C-I & Oca \\
\hline Falco sparverius & $\mathrm{C}-\mathrm{I}$ & Oce \\
\hline Ortalis canicolis & $\mathrm{O}$ & Per \\
\hline Chunga burmeisteri & $\mathrm{O}$ & Oca \\
\hline Vanellus chilensis & I & $\mathrm{Pe}$ \\
\hline Columba picazuro & G & Oca \\
\hline Columba maculosa & $\mathrm{G}$ & Per \\
\hline Zenaida auriculata & $\mathrm{G}$ & $\mathrm{Pe}$ \\
\hline Columbina picui & G & Per \\
\hline Leptotila verrauxi & G-I & Per \\
\hline Aratinga acuticaudata & F-G & $\mathrm{Per}$ \\
\hline Myiopsitta monachus & F-G & Per \\
\hline Coccyzus melacoryphus & I & Eest \\
\hline Tapera naevia & I & Ees \\
\hline Crotophaga ani & I & Oca \\
\hline Guira guira & C-I & Pel \\
\hline Tyto alba & $\mathrm{C}$ & Oca \\
\hline Otus choliba & I & Ein \\
\hline Glaucidium sp. & I & Ein \\
\hline Athene cunicularia & $\mathrm{I}-\mathrm{C}$ & $\mathrm{Pe}$ \\
\hline Strix rufipes & $\mathrm{C}$ & $\mathrm{Oca}$ \\
\hline Podager nacunda & I & Ees \\
\hline Caprimulgus longirostris & I & Ein \\
\hline Caprimulgus parvulus & I & $\mathrm{Pe}$ \\
\hline Hydropsalis brasiliana & I & Ees \\
\hline Chlorostilbon aureoventris & N-I & Ees \\
\hline Heliomaster furcifer & $\mathrm{N}-\mathrm{I}$ & Ees \\
\hline Nystalus maculatus & I & Per \\
\hline Melanerpes cactorum & I-F & $\mathrm{Per}$ \\
\hline Picoides mixtus & I-G & Per \\
\hline Colaptes melanochloros & I & $\mathrm{Pe}$ \\
\hline Colaptes campestris & I & Ees \\
\hline Drymornis bridgesii & I & Oca \\
\hline Lepidocolaptes & I & $\mathrm{Pe}$ \\
\hline
\end{tabular}


angustirostris

Upucerthia certhiodes

Furnarius rufus

Furnarius cristatus

Leptasthenura platensis

Synallaxis frontalis

Synallaxis albescens

Cranioleuca pyrrophia

Asthenes baeri

Phacellodomus sibilatrix

Coryphistera alaudina

Anumbius annumbi

Pseudoseisura lophotes

Taraba major

Thamnophilus

caerulescens

Rhinocrypta lanceolata Camptostoma obsoletum

Elaenia parvirostris

Serpophaga subcristata

Stigmatura budytoides

Euscarthmus meloryphus

Pyrocephalus rubinus

Agriornis murina

Xolmis irupero

Xolmis coronata

Neoxolmis rubetra

Knipolegus aterrimus

Knipolegus striaticeps

Myiarchus swainsoni

Pitangus sulfuratus

Empidonomus

aurantioatrocristatus

Sublegatus modestus

Hirundinea ferruginea

Machetornis rixosus

Hemitriccus

margaritaceiventer

Miyophobus fasciatus

Tyrannus savana

Pachyramphus

polychopterus

Phytotoma rutila

Phaeprogne tapera

Tachicyneta leucorrhoa

Progne modesta

Notiochelidon cyanoleuca

Troglodytes aedon

Polioptila dumicola

Turdus amaurochalinus

Turdus rufiventris

Mimus saturninus

Mimus triurus

Anthus correndera

Vireo olivaceus

Cyclarhis guajanensis

Parula pitiayumi

Geothlypis aequinoctalis

Euphonia chlorotica

Thraupis bonariensis

Saltator aurantiirostis

Paroaria coronata

Sporophila caerulescens

Coryphospingus cucullatus

Saltatricula multiculor

Sicalix flaveola

Sicalis luteola

Embernagra platensis

Poospiza melanoleuca

Poospiza nigrorufa

Poospiza torquata

Aimophila strigiceps

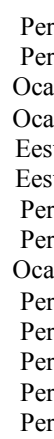

$\mathrm{Oca}^{1}$

$\mathrm{Oca}^{2}$

Per

Eest $^{1}$

Per

Eest

Eest

Oca

Per

Einv

$\mathrm{Oca}^{3}$

Per

Per

$\mathrm{Oca}^{2}$

Per

Eest

Oca $^{1}$

$\mathrm{Oca}^{1}$

Oca ${ }^{1}$

Einv ${ }^{1}$

Eest

Eest

$\mathrm{Oca}^{2}$

Per

Eest

Eest

Eest

Eest

Per

Per

Per

$\mathrm{Oca}^{1}$

Per

Einv

Per

Eest

Per

Oca

Eest

$\mathrm{Oca}^{1}$

Per

Per

Per

Eest

Per

Per

$\mathrm{Oca}^{1}$

Per

Oca $^{1}$

Per

Eest $^{1}$

Per

Per

$\begin{array}{lcc}\text { Zonotrichia capensis } & \text { G-I } & \text { Per } \\ \text { Sturnella superciliaris } & \text { G-I } & \text { Eest }^{1} \\ \text { Molothrus bonariensis } & \text { G-I } & \text { Per } \\ \text { Molothrus rufoaxillaris } & \text { G-I } & \text { Oca }^{1} \\ \text { Molothrus badius } & \text { G-I } & \text { Per } \\ \text { Carduelis magellanica } & \text { G } & \text { Eest }^{1}\end{array}$

Referencias: Gremio trófico: O: Omnívoro, I: Insectívoro, G: Granívoro, C; Carnívoro, Ñ: Carroñero, F: Frugívoro, N: Nectarívoro, H: Herbívoro. Estatus de residencia: Per: Permanente, Einv: Estacional invernal, Eest: Estacional estival, Oca: Ocasional. Superíndices ${ }^{1}$ : Permanente; ${ }^{2}$ : Estival, ${ }^{3}$. Invernal, según Nores (1996).

\section{Discusión}

El conjunto de aves registradas en los relictos de bosque al NW de la Mar Chiquita, representa alrededor del $30 \%$ de las especies que habitan el Chaco en toda su extensión (Bolivia, Paraguay y Brasil) (Short, 1975). En Argentina son escasos los relevamientos de aves en esta ecorregión; en la provincia de Córdoba, Dardanelli \& Nores (2001) citan 73 especies y Bucher et al. (2001) 87 en parches de Chaco y Espinal (éste aún más degradado). En Santiago del Estero Codesido \& Bilenca (2004) mencionan 96 especies. El número de entidades registrado en este trabajo (127) es superior, posiblemente por el mosaico determinado por una variedad de fisonomías y condiciones ecosistémicas (parches de bosque y pastizal, cultivos, proximidad de bañados y laguna, etc.) que allí se presentan.

En la composición específica se advierten elementos de interés. En primer lugar, cabe destacar la presencia de Harpyhaliaetus coronatus y Buteo polyosoma predadores de pequeños y medianos mamíferos. La primera es una rapaz de gran volumen que necesita amplia continuidad de hábitat chaqueño típico, ya que caza en cercanías o dentro del mismo; en adición se trata de una especie Vulnerable a nivel nacional (Collar et al., 1994). Ésta y la especie Ortalis canicollis, que estaría recuperándose en la provincia (Nores, 1996), resultaron más frecuentes en la condición de bosque maduro, con alto dosel arbóreo. Se constataron asimismo especies que frecuentan ambientes acuáticos (Nores \& Izurieta, 1980), tales como Bubulcus ibis, Casmerodius albus, Syrigma sibilatrix y Vanellus chilensis, por la proximidad de los Bañados del Río Dulce y la Laguna de Mar Chiquita, ubicados entre 10 a $20 \mathrm{~km}$ hacia el Este.

Como en otros sitios de bosque chaqueño, en nuestro estudio las insectívoras compusieron una elevada proporción de las especies (Capurro \& Bucher, 1986; Codesido \& Bilenca, 2004). Evidentemente, los insectos constituyen un recurso importante en este bosque con variados microhábitats en corteza, suelo, follaje y espacio aéreo (Caziani, 1996). La utilización de una variedad mayor de categorías alimenticias por las especies de residencia permanente se relacionaría con su capacidad 
adaptativa para aprovechar diversos recursos que proporciona la complejidad estructural del bosque.

En cuanto al estatus de residencia, las especies de presencia permanente fueron las mejor representadas. Codesido \& Bilenca (2004) obtienen porcentaje mayor (69\%) de éstas en el bosque de Santiago del Estero. En nuestro estudio un $24 \%$ de las "permanentes" citadas por Nores (1996) fueron registradas con baja frecuencia, sumando en la categoría "ocasional". Esta mengua en los encuentros de dichas especies sugiere disminución de su abundancia, en lo cual influiría la fragmentación y rápida pérdida de hábitat chaqueño, ocurridas en la última década en Córdoba (Kufner et al., 2005). La proporción de visitantes estivales triplicó a los invernales en relación con la mayor disponibilidad de recursos en general durante la faz húmeda en el Chaco (Codesido \& Bilenca, 2004).

El paisaje fragmentado de bosque rodeado por una matriz de mosaicos cultivados y vegetación secundaria, probablemente influyó en la presencia de especies de hábitats abiertos y antropizados. Así, habrían sido favorecidas aquéllas que duermen y nidifican en árboles frecuentemente en forma colonial (Ej.: Columba maculosa, Zenaida auriculata y Myiopsitta monacha). Estas granívoras y frugívoras presentaron elevados números y son consideradas plagas de la agricultura en Córdoba (Bucher \& Nores, 1988).

La región de estudio está relacionada con las Áreas importantes para la conservación de las aves en la Argentina (AICA) de la provincia de Córdoba (Di Giácomo, 2005) y la información que aporta la presente publicación será de utilidad para la planificación de estrategias tendientes a la conservación de dichas áreas.

Agradecimientos: La investigación fue parcialmente financiada por la SECyT de la Universidad Nacional de Córdoba. Especial reconocimiento al Biól. Gustavo Bruno por sus comentarios y aportes al manuscrito.

\section{Literatura citada}

Albarece E.A. \& Antelo C.M. 2000. Estudio preliminar de la avifauna residente en primavera en un sector del bosque chaqueño (Tucumán, Argentina). Acta Zoológica Lilloana, 45(2): 181-188. Tucumán.

Almazán-Núñez R.C. \& Navarro A.G. 2006. Avifauna de la subcuenca del río San Juan, Guerrero, México. Revista Mexicana de Biodiversidad. 77: 103-114. México.

Capurro H.A. \& Bucher E.H. 1986. Variación estacional en la comunidad de aves del bosque chaqueño de Chamical. Phycis (Buenos Aires), Secc. C. 44(106): 1-6.

Caziani S.M. 1996. Interacción plantas-aves dispersoras en un bosque chaqueño semiárido. Tesis Doctoral Universidad de Buenos Aires, Argentina.

Codesido M.A. \& Bilenca D.B. 2004. Variación Estacional de un Ensamble de Aves en un Bosque Subtropical Semiárido del Chaco Argentino. Biotrópica. 36(4): 544554.
Beissinger S.R., Steadman E.C., Wohlgenant T.W., Blate G., \& Zack S. 1996. Null models for assessing ecosystem conservation priorities: threatened birds as titres of threatened ecosystems in South America. Conserv. Biol. 10: 1343-1352.

Birdlife International (2006). www.birdlife.org.

Brooks D.M. 1997. Avian seasonality at a locality in the central Paraguayan Chaco. Hornero. 14: 193-203. Buenos Aires.

Bucher E.H., Costa Gorriz B. \& Leynaud G. 2001. Bird diversity and forest fragmentation in the semiarid espinal woodland of Córdoba, Argentina. Bol. Acad. Nac. Ciencias, Córdoba. 66: 117-124.

Bucher E.H. \& Nores M. 1988. Present status of birds in steppes and savannas of Northen and Central Argentina. ICBP Technical Publication $\mathrm{N}^{\circ} 7$.

Cabido M. \& Zak M. 1999. Vegetación del Norte de Córdoba. Secretaría de Agricultura, Ganadería y Recursos Renovables. Córdoba.

Cabrera A. \& Willink A. 1980. Biogeografía de América Latina. Serie de Biología. Monografía N 13. 117 pp. OEA, Washington.

Capurro H.A. \& Bucher E.H. 1988. Lista comentada de las aves del bosque chaqueño de Joaquín V. González, Salta, Argentina. Hornero. 13: 39-46. Buenos Aires.

Chani J.M., Lucero M.M., Marigliano N.L. \& Echevarría A.L. 1998. Lista comentada de aves de El Salvador, dapartamento Chuquisaca, Bolivia. Acta Zoológica Lilloana, 44(1): 191-217. Tucumán.

CITES. 2006 (Convention on International Trade in Endangered Species of Wild Flora and Fauna) www.cites.org.

Collar N.J., Crosby M.J. \& Stattersfield A.J. 1994. Birds to watch 2: the World List of Threatened Birds. Birdlife, Cambridge.

Dardanelli S. \& Nores M. 2001. Extinción y colonización de aves en fragmentos de bosque de la Provincia de Córdoba, Argentina. Bol. Acad. Nac. Ciencias, Córdoba. 66: 55-60.

Di Giacomo A.S. (editor) 2005. Áreas Importantes para la Conservación de las Aves en la Argentina. Sitios prioritarios para la conservación de la biodiversidad. Monografía de Aves Argentinas No 5:1-514. Asociación Ornitológica del Plata, Buenos Aires.

Kufner M., Giraudo L., Gavier G., Altrichter M., Tamburini D. \& Sironi M. 1998. Fauna de tetrápodos y sus biotopos en la Reserva La Quebrada, Río Ceballos, Córdoba. Acta Zoológica Lilloana. 44 (1): 177-184.

Kufner M., Tamburini D., Giraudo L. \& Briguera V. 2005. Conservación de mastofauna en fragmentos de Bosque Chaqueño en la región de Mar Chiquita, Córdoba, Argentina. Ecología Aplicada. 4(1,2): 53-58.

Leveau L.M. \& Leveau CM. 2004. Comunidades de aves en un gradiente urbano de la ciudad de Mar del Plata, Argentina. Hornero (Buenos Aires), jan./ago. 19(1): 1321. ISSN 0073-3407.

Mazar Barnett J. \& Pearman M. 2001. Lista comentada de las aves argentinas. Asociación Ornitológica del Plata. Birdlife International. Lynx Edicions. Barcelona.

Miatello R. 1994. Vertebrados en vías de extinción de la provincia de Córdoba. Tras la Huella. Recursos Naturales. 3(11): 36-39. Córdoba.

Morello J. \& Matteucci S. 1999. El difícil camino al manejo rural sostenible en la Argentina. 41 - 54. En: S. 
Matteucci, O. Solbrig, J. Morello \& G. Halffter (Eds.). Biodiversidad y uso de la tierra. Conceptos y ejemplos de Latinoamérica. Eudeba. B. Aires.

Narosky T. \& Izurieta D. 2003. Aves de Argentina y Uruguay: guía para la identificación. Asociación Ornitológica del Plata. Birdlife International. $15^{\text {a }}$ Edición. Buenos Aires. Vázquez Mazzini Editores.

Nores M. \& Izurieta D. 1980. Aves de ambientes acuáticos de Córdoba y centro de Argentina. Sec. Est. Agr. Gan. Córdoba.

Nores M. 1996. Avifauna de la provincia de Córdoba. 255 338. En: I. di Tada y E. Bucher (Eds.). Biodiversidad de la Provincia de Córdoba. Vol. 1. Fauna. Universidad Nacional de Río Cuarto. Río Cuarto.

Olrog C.C. 1963. Lista y distribución de las aves argentinas. Opera Lilloana, 9. Tucumán.

Sick H. 1985. Ornitología brasileira, uma introduçào. Brasilia, Editora Universidade de Brasilia. Vol I y II. : 598.
Short L.L. 1975. A zoogeographic análisis of the South American Chaco avifauna. Bulletin of the American Museum of Natural History. 154 (3): 166-352. New York.

Torres R. \& Michelutti P. 2001. Nuevos registros de aves escasas en la región central de Argentina. Nótulas Faunísticas (Segunda Serie). 1: 1-5.

Torres R. \& Michelutti P. 2005. Bañados del Río Dulce y Laguna de Mar Chiquita Pp: 134-137, En: Di Giácomo A.S. (Ed.) Áreas Importantes para la Conservación de las Aves en la Argentina. Temas de Naturaleza y Conservación. 5: 1-514. Aves Argentinas/Asociación Ornitológica del Plata, Buenos Aires.

Zak M. \& Cabido M. 2002. Spatial patterns of the Chaco vegetation of central Argentina: Integration of remote sensing and phytosociology. Applied Vegetation Science. 5: 213-226.

${ }^{2}$ CERNAR y ${ }^{1}$ Cátedra Diversidad Animal II, FCEFyN, UNC. Avenida Vélez Sarsfield 299, 5000, Córdoba, Argentina.

Direcciones electrónicas de contacto: lgiraudo@com.uncor.edu; bkufner@com.uncor.edu 